\title{
Monotherapy for partial epilepsy: focus on levetiracetam
}

\author{
Antonio Gambardella ${ }^{1,2}$ \\ Angelo Labate ${ }^{1,2}$ \\ Eleonora Colosimo' \\ Roberta Ambrosio' \\ Aldo Quattrone ${ }^{1,2}$ \\ 'Institute of Neurology, University \\ Magna Græcia, Catanzaro, Italy; \\ ${ }^{2}$ Institute of Neurological Sciences, \\ National Research Council, Piano \\ Lago di Mangone, Cosenza, Italy
}

\begin{abstract}
Levetiracetam (LEV), the S-enantiomer of alpha-ethyl-2-oxo-1-pyrollidine acetamide, is a recently licensed antiepileptic drug (AED) for adjunctive therapy of partial seizures. Its mechanism of action is uncertain but it exhibits a unique profile of anticonvulsant activity in models of chronic epilepsy. Five randomized, double-blind, placebo-controlled trials enrolling adult or pediatric patients with refractory partial epilepsy have demonstrated the efficacy of LEV as adjunctive therapy, with a responder rate ( $\geq 50 \%$ reduction in seizure frequency) of $28 \%-45 \%$. Long-term efficacy studies suggest retention rates of $60 \%$ after one year, with $13 \%$ of patients seizure-free for 6 months of the study and $8 \%$ seizure-free for 1 year. More recent studies illustrated successful conversion to monotherapy in patients with refractory epilepsy, and its effectiveness as a single agent in partial epilepsy. LEV has also efficacy in generalized epilepsies. Adverse effects of LEV, including somnolence, lethargy, and dizziness, are generally mild and their occurrence rate seems to be not significantly different from that observed in placebo groups. LEV also has no clinically significant pharmacokinetic interactions with other AEDs, or with commonly prescribed medications. The combination of effective antiepileptic properties with a relatively mild adverse effect profile makes LEV an attractive therapy for partial seizures.
\end{abstract}

Keywords: levetiracetam, partial epilepsy, antiepileptic drugs

\section{Introduction}

Levetiracetam (LEV), (S)- $\alpha$-ethyl-2-oxo-pyrrolidine acetamide analog of piracetam (Shorvon 2001), is a new anticonvulsant agent with a favorable tolerability profile and a low potential for drug interactions (Dooley and Plosker 2000). LEV was synthesized in the early 1980s during a follow-up chemical program aimed at identifying a second-generation nootropic drug, and initial pharmacologic studies with LEV explored its ability to facilitate cholinergic neurotransmission (Klitgaard 2001). In 1991, pivotal clinical studies were initiated in epilepsy patients as adjunctive therapy in refractory partial onset seizures. In November 1999, the FDA approved LEV as a new antiepileptic drug (AED).

LEV has demonstrated efficacy and a favorable tolerability profile as adjunctive for partial seizures in adult and pediatric patients (Hovinga 2001; Shorvon and van Rijckevorsel). Moreover, there has been increasing evidence that LEV may also be useful in patients with generalized absence or myoclonic seizures, and in patients with Lennox-Gastaut syndrome (Kasteleijn-Nolst et al 1996; Labate et al 2006). LEV has become one of the most frequently prescribed new drugs for the treatment of partial seizures. It offers several advantages over traditional therapy, including twice daily dosing, a wide margin of safety with no requirements for serum drug concentration monitoring, and no interactions with other anticonvulsants. In addition, LEV appears to be well tolerated by most patients and may have less adverse effects on cognitive function than traditional agents (Dooley and Plosker 2000). 
This advantageous pharmacologic profile makes LEV an attractive first line or adjunctive therapy for epileptic seizures. This review focuses on the experience and use of LEV in partial epilepsy.

\section{Experimental studies}

The mechanism for the anticonvulsant effect of LEV still remains elusive. LEV is not chemically related to other anticonvulsants and its mechanism of action seems to be unrelated to known mechanisms of neurotransmission. Indeed, unlike other AEDs, LEV has no effect in the two classic rodent models for AEDs, the maximal electroshock seizure and the pentylenetetrazol (Gower et al 1992; Löscher and Hönack 1993). Moreover, the drug does not bind to receptors associated with excitatory or inhibitory neurotransmitters including $\gamma$-aminobutyric acid (GABA), glutamate, glycine, adenosine; it also has no effect on sodium or T-type calcium channel function, and does not affect GABA transaminase or glutamic acid decarboxylase (GAD) activity or second messenger systems (cyclic adenosine monophosphate) or protein kinase C (Löscher and Hönack 1993). Conversely, LEV seems to partially inhibit N-type high-voltage-activated $\mathrm{Ca}^{2+}$ currents and reduces the $\mathrm{Ca}^{2+}$ release from intraneuronal stores (Gower et al 1995; Klitgaard et al 1998; Löscher et al 1998; Rigo et al 2000; Niezpodziany et al 2001; Zona et al 2001). It also reverses inhibition of GABA and glycine gated currents induced by negative allosteric modulators (Rigo et al 2000), and effects voltage gated potassium channel conductance (Madeja et al 2001). LEV also has a specific stereoselective binding site in the CNS at the synaptic vesicle protein 2A (SV2A) (Dooley and Plosker 2000; Rigo et al 2000), and cannot be displaced from this site by other classic AEDs such as carbamazepine, phenytoin, valproate, and phenobarbital, although ethosuximide does show binding affinity. LEV has no binding to membranes outside of the CNS.

LEV has very marked protection against seizures in audiogenic mice, mice kindled with corneal electroshock or PTZ, and amygdaloid kindled rats (Klitgaard et al 1998; Löscher et al 1998). It protects against spontaneous spike and wave discharges in the GAERS model and in pilocarpine or kainic acid induced focal seizures in rats (Klitgaard et al 1998; Löscher et al 1998). The extent of the antiepileptic efficacy in the audiogenic seizure model in mice was found to be correlated with the affinity for the binding site of a series of S-homologues of LEV (Noyer et al 1995). The dose-dependent ability of LEV to inhibit the development of kindling suggests a potential antiepileptogenic effect as well (Löscher et al 1998). LEV is the most effective of any of the pyrrolidone drugs in these epilepsy models. Its R-enantiomer has no antiepileptic activity.

The dose at which toxic effects on the rotarod test are produced is much higher than the effective antiseizure dose in both the GAERS model and the corneally kindled mice. The safety margin of LEV in these models is much greater than for other drugs (Harden 2001). In acute and chronic toxicity studies in animals, LEV shows generally low toxicity. Oral doses up to $5000 \mathrm{mg} / \mathrm{kg}$ acutely (maximum tested dose) are not lethal in mice and rats. LEV has not displayed any teratogenic, mutagenic, or carcinogenic properties (French et al 2001; Harden 2001).

\section{Clinical pharmacokinetics}

The pharmacokinetic properties of LEV have been studied in healthy adult volunteers, patients with epilepsy, and special populations, including pediatric and elderly patients and patients with renal or hepatic insufficiency (Pellock et al 2001; Radtke 2001). LEV is highly soluble in water. It is formulated for clinical use as 250-, 500-, and 1000-mg film-coated tablets. LEV is rapidly and almost completely absorbed after oral administration of doses ranging from $250 \mathrm{mg}$ to $5000 \mathrm{mg}$, with peak serum concentrations occurring approximately 1 hour after a dose and steady state concentrations reached within 48 hours. Absorption of LEV is unaffected by the presence of food or antacids, although the rate of absorption may be slowed. It has been now produced an IV formulation of LEV whose infusion is bioequivalent to oral tablets and is well tolerated after $15 \mathrm{~min}$ and $5 \mathrm{~min}$ IV infusion in healthy subjects (Ramael et al 2006).

LEV exhibits minimal protein binding $(<10 \%)$ and has a volume of distribution of $0.5-0.7 \mathrm{~L} / \mathrm{kg}$ in adults, similar to the volume of distribution of intracellular and extracellular water. In addition, LEV exhibits linear, dose proportional, kinetics, with low intrasubject and intersubject variability, and a halflife of 6-8 hours (Pellock et al 2001; Radtke 2001).

LEV is eliminated through renal excretion, primarily as unchanged drug. LEV does not undergo hepatic metabolism (Nicolas et al 1999), even if a minor percentage undergoes hepatic metabolism via enzymatic hydrolysis and hydroxylation to inactive byproducts. Clearance is rapid, so that within 48 hours approximately $93 \%$ of an oral dose is eliminated. The elimination half-life of LEV in healthy adults ranges from 6 to 8 hours, in children is 5-7 hours and in elderly between 10 and 11 hours, regardless of dosage or frequency of administration. The prolonged elimination half life of LEV in the elderly is likely attributable to the age related decline in renal function. Average total body 
clearance is $0.96 \mathrm{~mL} / \mathrm{min} / \mathrm{kg}$ in adults, with a renal clearance of $0.6 \mathrm{ml} / \mathrm{min} / \mathrm{kg}$. After single oral dose administration of $20 \mathrm{mg} / \mathrm{kg} \mathrm{LEV}$ in children between 6 and 12 years of age, total body clearance was about $30 \%-40 \%$ higher than in adults. Renal clearance of LEV is directly proportional to creatinine clearance. In adult patients with severe renal impairment (creatinine clearance $<30 \mathrm{~mL} / \mathrm{min}$ ), LEV clearance is reduced by approximately $60 \%$ (Radtke 2001). Clearance of LEV is significantly reduced in patients with severe hepatic impairment and concomitant renal impairment (hepatorenal syndrome). No differences are seen in patients with mild to moderate hepatic impairment (Pellock et al 2001).

The recommended dosing regimen for LEV as add on therapy is twice daily doses of $500-1500 \mathrm{mg}$, for a total daily dosage of between $1000 \mathrm{mg}$ and $3000 \mathrm{mg}$. Higher doses have been studied, but with little evidence of added effectiveness. The initial starting dose of $1000 \mathrm{mg} /$ day has been shown to be clinically effective, but if sufficient seizure control is not obtained, doses can be increased up to $3000 \mathrm{mg} /$ day. LEV has been shown to be effective as early as the first day of therapy, and this rapid effect is complemented by a sustained efficacy (French and Arrigo 2005). In patients with renal impairment, doses should be reduced in accordance with creatinine clearance (Keppra 2000a, b). Presently, there are no sufficient data to recommend treatment with LEV during pregnancy. In patients withdrawn from LEV, a gradual tapering of $1000 \mathrm{mg}$ every 1-2 weeks has been successful and has not resulted in withdrawal seizures.

\section{Drug interactions}

Because of its advantageous pharmacokinetic, LEV does not appear to interact with other AEDs (Nicolas et al 1999), and the overall pharmacokinetic parameters of LEV during polytherapy with AEDs are comparable to those of subjects receiving LEV alone. The pharmacokinetic profile of LEV is not influenced by phenytoin, phenobarbital, primidone, carbamazepine, valproic acid, lamotrigine, gabapentin, digoxin, oral contraceptives ethinylestradiol, and warfarin (Browne et al 2000; French et al 2001; Levy et al 2001; Radtke 2001; Shorvon and van Rijckevorsel 2002). Similarly, the addition of LEV does not significantly alter serum concentrations of all these drugs. Pooled analysis confirmed these findings (Gidal et al 2005). The possible interaction between LEV and tiagabine, topiramate, and zonisamide has not been investigated. A recent experimental study illustrated a pharmacokinetic contribution other than pharmacodynamic interaction between LEV and felbamate (Luszczki et al 2007).

\section{Side effects}

The most commonly reported adverse effects during clinical trials with LEV in adults were primarily related to the CNS and included somnolence (15\% of patients), asthenia (15\%), headache (14\%), infection (13\%), dizziness ( $9 \%)$, and ataxia (3\%) (Ben-Menachem and Falter 2000; Cereghino et al 2000; Shorvon et al 2000). These adverse effects were seen most frequently in the first month of therapy and typically lessened or resolved with continued treatment. In the pooled analysis, there was no evidence of a dose dependent relation within the recommended dose range of 1000-3000 mg/day (Gidal et al 2005). Patients receiving LEV also reported a slightly higher incidence of symptoms of upper respiratory infection, which was not associated with leucopenia or dose reduction. In clinical trials, from one to $4 \%$ of patients have withdrawn because of these effects (Ben-Menachem and Falter 2000; Cereghino et al 2000; Shorvon et al 2000; Tsai et al 2006). Similar adverse effects, but higher percentages, were reported in pediatric populations (Glauser et al 2002, 2006).

In pre-marketing studies of $\mathrm{LEV}$, up to $13 \%$ of patients have experienced adverse neuropsychiatric symptoms. In most of these patients, the symptoms have been mild, including agitation, hostility, apathy, anxiety, emotional lability, and depression. Nonetheless, about $1 \%$ of pediatric or adult patients have experienced serious neuropsychiatric symptoms including hallucinations, suicidal ideations, or psychosis, after beginning LEV (Kossoff et al 2001; Mula et al 2003). There was a significant association between psychiatric adverse events and previous history of febrile convulsions or status epilepticus, while a past personal or family history of psychiatric disorders was more important in predicting the features of psychiatric adverse events rather than their occurrence. Moreover, psychiatric adverse events were not related to the starting dose, titration schedule of LEV or the rate of seizure freedom. In these reports, symptoms occurred mostly within the first month of therapy, but they could develop at any time during treatment. Dose reduction or discontinuation has led to resolution of symptoms in the cases reported. Overall, these studies illustrated that a close clinical monitoring with regard to psychiatric adverse events is related to the psychiatric profile of the patient (Kossoff et al 2001; Mula et al 2003). Conversely, LEV has no major adverse effects on cognitive function (Neyens et al 1995).

\section{Clinical antiepileptic effect Add on therapy in partial epilepsy}

The efficacy of LEV as add on therapy has been assessed in 5 prospective, double blind, placebo controlled trials in 
patients with uncontrolled partial seizures (Ben-Menachem and Falter 2000; Cereghino et al 2000; Shorvon et al 2000; Glauser et al 2006; Tsai et al 2006). Four of these studies enrolled adult patients with at least 2 refractory partial seizures per 4 weeks (Ben-Menachem and Falter 2000; Cereghino et al 2000; Shorvon et al 2000; Tsai et al 2006). The fifth study was carried out in children aged 4-16 years (Glauser et al 2006). Doses of LEV evaluated in adults included 1000, 2000, and $3000 \mathrm{mg} /$ day given in twice-daily regimens. Dose titration was of 4 weeks, followed by 12-14 weeks of maintenance. At all dosages evaluated in these three studies, LEV was significantly more effective than placebo. The median percentage reduction from baseline was $32.5 \%$ for patients receiving LEV compared with $7 \%$ for those receiving placebo $(\mathrm{p}<0.001)$. During the evaluation period, the responder rate, that is the proportion of patients experiencing a $50 \%$ or greater reduction in seizure frequency compared with baseline, was 27.7\% (54/195), 31.6\% (30/95), and $41.3 \%(111 / 269)$ for patients receiving 1000,2000 , and $3000 \mathrm{mg} /$ day respectively, compared with $12.6 \%$ (38/301) of patients who received placebo $(\mathrm{p}>0.001$, all doses versus placebo). The percentage of patients experiencing a $75 \%$ or greater reduction in seizures was $11.8 \%$ (23/195), 16.8\% (16/95), and $22.3 \%$ (60/269) of patients receiving $1000 \mathrm{mg}$, $2000 \mathrm{mg}$, and $3000 \mathrm{mg}$ of LEV respectively, compared with $3.3 \%(10 / 301)$ of placebo treated patients $(\mathrm{p}<0.001$, all doses versus placebo). In addition, $5.7 \%(32 / 559)$ of patients treated with LEV became seizure free, compared with $0.6 \%(2 / 301)$ in the placebo group ( $\mathrm{p}<0.001)$ (Cereghino et al 2000).

A statistically significant reduction in seizure frequency for all different subtypes of partial seizures (simple partial, complex partial, and secondarily generalized seizures) was found with LEV treatment. In addition a pooled analysis derived from the three studies in adults demonstrates a specific independent reduction of secondary generalized seizures (median percentage reduction: 68.5\%) (Leppik et al 2003).

A large multicenter, randomized, placebo-controlled trial illustrated that LEV as adjunctive therapy administered at 60 $\mathrm{mg} / \mathrm{kg} /$ day is also efficacious and well tolerated in children with drug-resistant partial seizures (Glauser et al 2006). During the treatment period, 198 patients received either placebo or LEV add-on therapy and were up-titrated to a target dose of $60 \mathrm{mg} / \mathrm{kg} / \mathrm{day}$. There was a significant $(26.8 \%$; $\mathrm{p}<0.0002 ; 95 \%$ CI $14.0 \%-37.6 \%$ ) reduction in partial onset seizure frequency per week for LEV adjunctive therapy over placebo adjunctive therapy. A $50 \%$ or greater reduction of partial seizure frequency per week was attained in $44.6 \%$ of the LEV group (45/101 patients), compared with $19.6 \%$ (19/97 patients) receiving placebo $(\mathrm{p}=0.0002)$.

\section{Monotherapy}

Although LEV is well tolerated with a favorable pharmacokinetic profile, few works demonstrated successful conversion to monotherapy in patients with refractory epilepsy, and few studies with small number of patients demonstrate its effectiveness as a single agent in partial epilepsy (Ben-Menachem and Falter 2000; Alsaadi et al 2005; Brodie et al 2007). In a multicenter double-blind, responder selected study (BenMenachem and Falter 2000), it was evaluated the efficacy and tolerability of LEV monotherapy in selected patients with refractory focal epilepsy: in the LEV monotherapy group the median percent reduction in partial seizure frequency compared with baseline was $73.8 \%$ with a responder rate of $59.2 \%$.

In an open study, LEV was efficacious as monotherapy in 46 patients with newly diagnosed naïve epilepsy or with chronic difficult to control epilepsy who were followed for 1 year (Alsaadi et al 2005). In this study, the majority (82\%) of the patients remained on LEV for at least 1 year with more than $50 \%$ of patients remaining seizure free. Moreover, a recent randomized double-blind trial involving 579 patients comparing LEV with controlled-release carbamazepine illustrated that both AEDs have produced equivalent seizure freedom rates in newly diagnosed epilepsy at optimal dosing in a setting mimicking clinical practice (Brodie et al 2007). Importantly, no other newer AED has been shown to be equivalent to an older generation AED (Brodie et al 2007). Furthermore, long-term evaluation of the patients enrolled in these trials, as well as several others, suggests that LEV is both efficacious and well tolerated by most patients. Based on compiled results, retention rates are estimated to be $60 \%$ at 1 year and $32 \%$ at 5 years (Krakow et al 2001).

\section{IV therapy}

LEV IV infusion is bioequivalent to oral tablets and is well tolerated after 15-min (2000-4000) and 5-min (1500-2500) IV infusions in healthy subjects (Ramael et al 2006a, b). Among the newer agents LEV has been the first to be approved for IV application. The results of a small, multicenter, open-label study (Baulac et al 2007) suggest that a 15-min infusion (500-1500 mg, bid) is well tolerated in patients with partial onset seizures when administered over a 4-day period. The observed adverse events were mild to moderate and the most frequently reported ones were headache and fatigue. None of the subjects discontinued because of 
adverse events and no serious adverse events were reported. These results support dosing flexibility and easy conversion from oral to IV LEV, and back, in patients with partial-onset seizures temporarily unable to take the drug orally.

\section{Conclusions}

LEV is a novel antiepileptic drug which has been approved as adjunctive treatment for adults with partial onset seizures. Its effectiveness was established in five multicenter, well-controlled pivotal trials. In addition, LEV is well tolerated with a favorable pharmacokinetic profile that includes minimal protein binding, lack of hepatic metabolism, and twice a day dosing. These features and others make it ideal for use as monotherapy (French et al 2004a, b). The majority of studies on LEV monotherapy effect in focal epilepsies in children, in adults, and in the elderly are based on retrospective evaluation of small series of patients with a short-term follow up; only one study compares LEV to an traditional AED; more additional randomized double-blind monotherapy trials are needed to confirm these findings.

\section{Disclosures}

The author has been a speaker at meetings organized by UCB Pharma.

\section{References}

Alsaadi TM, Shatzel A, Marquez AV, et al. 2005. Clinical experience of levetiracetam monotherapy for adults with epilepsy: 1-year follow-up study. Seizure, 14:139-42.

Baulac M, Brodie MJ, Christian EE, et al. 2007. Levetiracetam intravenous infusion as as alternative to oral dosing in patients with partial-onset seizures. Epilepsia, 48:589-92.

Ben-Menachem E, Falter U. 2000. Efficacy and tolerability of levetiracetam $3000 \mathrm{mg} / \mathrm{d}$ in patients with refractory partial seizures: a multicenter, double-blind, responder-selected study evaluating monotherapy. Epilepsia, 41:1276-83.

Brodie MJ, Perucca E., Ryvilin P., et al. 2007. Comparison of levetiracetam and controlled release carbamazepine in newly diagnosed epilepsy. Neurology, 68:402-8.

Browne TR, Szabo GK, Leppik IE, et al. 2000. Absence of pharmacokinetic drug interaction of levetiracetam with phenytoin in patients with epilepsy determined by new technique. J Clin Pharmacol, 40:590-5.

Cereghino JJ, Biton V, Abou-Khalil B, et al. 2000. Levetiracetam for partial seizures: results of a double-blind, randomized clinical trial Neurology, 55:236-42.

Dooley M, Plosker GL. 2000. Levetiracetam: a review of its adjunctive use in the management of partial onset seizures. Drugs, 60:871-93.

French J, Arrigo C. 2005. Rapid onset of action of levetiracetam in refractory epilepsy patients. Epilepsia, 46:324-6.

French J, Edrich P, Cramer JA. 2001. A systematic review of the safety profile of levetiracetam: a new antiepileptic drug. Epilepsy Res, 47:77-90.

French JA, Kanner AM, Bautista J, et al. 2004a. Efficacy and tolerability of the new antiepileptic drugs I: treatment of new onset epilepsy. Neurology, 62:1252-60
French JA, Kanner AM, Bautista J, et al. 2004b. Efficacy and tolerability of the new antiepileptic drugs II: treatment of refractory epilepsy. Neurology, 62:1261-73

Gidal BE, Baltès E, Otoul C, et al. 2005. Effect of levetiracetam on the pharmacokinetics of adjunctive antiepileptic drugs: a pooled analysis of data from randomized clinical trials. Epilepsy Res, 64:1-11.

Glauser TA, Ayala R, Elterman RD, et al. 2006. Double-blind, randomized, placebo-controlled trial of adjunctive levetiracetam in pediatric partial seizures. Neurology, 66:1654-60

Glauser TA, Pellock JM, Bebin EM, et al. 2002. Efficacy and safety of levetiracetam in children with partial seizures: an open-label trial. Epilepsia, 43:518-24

Gower AJ, Hirsch E, Boehrer A, et al. 1995. Effects of levetiracetam, a novel antiepileptic drug, on convulsant activity in two genetic rat models of epilepsy. Epilepsy Res, 22:207-13.

Gower AJ, Noyer M, Verloes R, et al. 1992. UCB L059, a novel anticonvulsant drug: pharmacological profile in animals. Eur J Pharmacol, 222:193-203

Harden C. 2001. Safety profile of levetiracetam. Epilepsia, 42(Suppl 4):36-9.

Hovinga CA. 2001. Levetiracetam: a novel antiepileptic drug. Pharmacotherapy, 21:1375-88.

Kasteleijn-Nolst Trenité DGA, Marescaux C, Stodieck S, et al. 1996. Photosensitive epilepsy: a model to study the effects of antiepileptic drugs. Evaluation of the piracetam analogue, levetiracetam. Epilepsy Res, 25:225-30

$\mathrm{Keppra}^{\mathrm{TM}}(250 \mathrm{mg}, 500 \mathrm{mg}, 1000 \mathrm{mg}) .2000 \mathrm{~b}$. Summary of product characteristics. Brussels, Belgium: UCB SA. June 2000

Keppra $^{\mathrm{TM}}$ levetiracetam 250, 500,750 mg tablets. 2000a. Prescribing information. Brussels, Belgium: UCB Pharma.

Klitgaard H, Matagne A, Gobert J, et al. 1998. Evidence for a unique profile of levetiracetam in rodent models of seizures and epilepsy. Eur $J$ Pharmacol, 353:191-206.

Klitgaard H. 2001. Levetiracetam: the preclinical profile of a new class of antiepileptic drugs? Epilepsia, 42(Suppl 4):13-18.

Kossoff EH, Bergey GK, Freeman JM, et al. 2001. Levetiracetam psychosis in children with epilepsy. Epilepsia, 42:1611-3.

Krakow D, Walker W, Otoul C, et al. 2001. Long-term continuation of levetiracetam in patients with refractory epilepsy. Neurology, 56:1772-4.

Labate A, Colosimo E, Gambardella A, et al. 2006. Levetiracetam in patients with generalised epilepsy and myoclonic seizures: an open label study. Seizure, 15:214-8

Leppik IE, Biton V, Sander JW, et al. 2003. Levetiracetam and partial seizure subtypes: pooled data from three randomized, placebo-controlled trials. Epilepsia, 44:1585-7.

Levy RH, Ragueneau-Majlessi I, Baltes E. 2001. Repeated administration of the novel antiepileptic agent levetiracetam does not alter digoxin pharmacokinetics and pharmacodynamics in healthy volunteers. Epilepsy Res, 46:93-9.

Löscher W, Hönack D, Rundfeldt C. 1998. Antiepileptogenic effects of the novel anticonvulsant levetiracetam (ucb L059) in the kindling model of temporal lobe epilepsy. J Pharmacol Exp Ther, 284:474-9.

Löscher W, Hönack D. 1993. Profile of ucb L059, a novel anticonvulsant drug, in models of partial and generalised epilepsy in mice and rats. Eur J Pharmacol, 232:147-58.

Luszczki JJ, Andres-Mach MM, Ratnaraj N, et al. 2007. Levetiracetam and felbamate interact both pharmacodynamically and pharmacokinetically: an isobolographic analysis in the mouse maximal electroshock model. Epilepsia, 48:806-15.

Madeja M, Margineanou D, Klitgaard H. 2001. Effect of levetiracetam on voltage-gated, potassium channels: a novel anti-epileptic mechanism? Epilepsia, 42(suppl 2):19.

Mula M, Trimble MR, Yuen A, et al. 2003. Psychiatric adverse events during levetiracetam therapy. Neurology; 61:704-6.

Neyens LGJ, Alpherts WCJ, Aldenkamp AP. 1995. Cognitive effects of a new pyrrolidine derivative (levetiracetam) in patients with epilepsy. Prog Neuro-Psychopharmacol Biol Psychiatry; 19:411-9. 
Nicolas JM, Collart P, Gerin B, et al. 1999. In vitro evaluation of potential drug interactions with levetiracetam, a new antiepileptic agent. Drug Metab Dispos, 27:250-4.

Niespodziany I, Klitgaard H, Margineanu D-G. 2001. Levetiracetam inhibits the higher-voltage-activated $\mathrm{Ca}^{++}$current in pyramidal neurons of rat hippocampal slices. Neurosci Lett, 306:5-8.

Noyer M, Gillard M, Matagne A, et al. 1995. The novel antiepileptic drug levetiracetam (ucb L059) appears to act via a specific binding site in CNS membranes. Eur J Pharmacol, 286:137-46.

Pellock JM, Glauser TA, Bebin EM, et al. 2001. Pharmacokinetic study of levetiracetam in children. Epilepsia, 42:1574-9.

Radtke RA. 2001. Pharmacokinetics of levetiracetam. Epilepsia, 42(Suppl 4):24-7.

Ramael S, Daoust A, Otoul C, et al. 2006b. Levetiracetam intravenous infusion: a randomized, placebo-controlled safety and pharmacokinetic study. Epilepsia. 47:1128-35.

Ramael S, De Smedt F, Toublanc N, et al. 2006. Single dose bioavailability of levetiracetam intravenous infusion relative to oral tablets and multiple-dose pharmacokinetics and tolerability of levetiracetam intravenous infusion compared with placebo in healthy subjects. Clin Ther, 28:734-44
Ramael S, De Smedt F, Toublanc N, et al. 2006a. Single-dose bioavalability of levetiravetam intravenous infusion relative to oral tablets and multiple-dose pharmacokinetics and tolerability of levetiracetam intravenous infusion compared with placebo in healthy subjects. Clinical Therapeutics, 28:734-44.

Rigo J-M, Nguyen L, Belachew S, et al. 2000. Levetiracetam: novel modulation of ionotropic inhibitory receptors. Epilepsia, 41(Suppl 7):35.

Shorvon SD, Lowenthal A, Janz D, et al. 2000. Multicenter double-blind, randomized, placebo-controlled trial of levetiracetam as add-on therapy in patients with refractory partial seizures. Epilepsia, 41:1179-86.

Shorvon SD, van Rijckevorsel K. 2002. A new antiepileptic drug [editorial]. J Neurol Neurosurg Psychiatry, 72:426-8.

Shorvon SD. 2001. Pyrrolidone derivatives. Lancet, 200:1885-92.

Tsai JJ, Yen DJ, Hsih MS, et al. 2006. Efficacy and safety of levetiracetam (up to $2000 \mathrm{mg} /$ day) in Taiwanese patients with refractory partial seizures: a multicenter, randomized, double-blind, placebo-controlled study. Epilepsia, 47:72-81.

Zona C, Niespodziany I, Marchetti C, et al. 2001. Levetiracetam does not modulate neuronal voltage-gated $\mathrm{Na}+$ and $\mathrm{T}$-type $\mathrm{Ca} 2+$ currents. Seizure, 10:279-86. 